\title{
HISTOPLASMOMA COMO LESÃO ISOLADA DO SISTEMA NERVOSO CENTRAL EM PACIENTE IMUNOCOMPETENTE
}

\author{
Emerson L. Gasparetto ${ }^{1}$, Arnolfo de Carvalho Neto², James Alberton ${ }^{3}$, Taisa Davaus ${ }^{3}$, \\ Mara Albonei D. Pianovski ${ }^{4}$, Érica Yamauchi ${ }^{5}$, Luiz F. Bleggi Torres $^{6}$
}

\begin{abstract}
RESUMO - O acometimento cerebral pela histoplasmose é raro, ocorrendo mais comumente sob a form a de doença disseminada. Raramente, a doença pode ocorrer sob a forma de histoplasmomas, que simulam tumores do sistema nervoso central. Mais raro ainda é a ocorrência de histoplasmomas em pacientes imunocompetentes como única manifestação desta infecção. Neste relato é apresentado um paciente masculino de 13 anos com cefaléia, vômitos, redução da acuidade visual e auditiva à esquerda e hemiparesia à direita. A ressonância magnética mostrou lesão expansiva com impregnação anelar de contraste, localizada na região talâmica, hipotalâmica e quiasmática à esquerda. Foi realizada biópsia estereotáxica e a avaliação histológica do material definiu o diagnóstico de histoplamose. Iniciou-se tratamento com fluconazol, com melhora clínica importante após 6 meses do início do tratamento.
\end{abstract}

PALAVRAS-CHAVE: histoplasmose, sistema nervoso central, ressonância magnética.

\begin{abstract}
Histoplasmoma as isolated central nervous system lesion in an immunocompetent patient
ABSTRACT - The cerebral lesions are uncommon in patients with histoplasmosis, occurring more frequently in the disseminated form of the disease. Rarely, the disease may present as a histoplasmoma, simulating a neoplastic lesion. The histoplasmoma as the only manifestation of this infection in immunocompetent patients is even rarer. This case report describes a 13 year-old male patient with headache, vomit, low visual acuity and auditive deficit on the left, and paresis on the right. The magnetic resonance image showed an expansible lesion in the thalamic, hypothalamic, and chiasmatic regions, which showed ring enhancement. The stereotactic biopsy was perf o rmed and the histological diagnosis of histoplasmosis was defined. The treatment was initiated with fluconazole. The patient showed important clinical improvement after 6 months.
\end{abstract}

KEY WORDS: histoplasmosis, central nervous system, magnetic resonance

A histoplasmose é doença causada pelo fungo Histoplasma capsulatum, que é endêmico em determinadas regiões do mundo, dentre elas o Brasil. Grandes inóculos do fungo, dispersos no ar, favorecem a infecção, que pode ser dividida em: 1. doença progressiva grave, em geral disseminada, acometendo indivíduos com comprometimento da imunidade; 2 . doença localizada, em geral autolimitada, tendendo a ocorrer em surtos e acometendo indivíduos imunocompetentes ${ }^{1,2}$. $\mathrm{O}$ acometimento do sistema nervoso central (SNC) é inco- mum, sendo mais freqüente em pacientes imunodeprimidos, sob a forma de doença disseminada. A ocorrência da infecção cerebral sob a forma tumoral (histoplasmoma) é rara, e em geral representa manifestação da doença disseminada. Nestes casos, o diagnóstico diferencial com neoplasias cerebrais através de dados clínicos e de imagem muitas vezes é difícil, tornando a biópsia da lesão inevitável para que se obtenha a definição diagnóstica ${ }^{1,3,4}$.

Relatamos um caso de histoplasmose com aco-

\footnotetext{
Disciplina de Radiologia Médica, Departamento de Pediatria e Departamento de Patologia da Universidade Federal do Paraná, Curitiba PR, Brasil (UFPR): ${ }^{1}$ Médico Residente do Serviço de Radiologia Médica e Diagnóstico por Imagem, Hospital de Clínicas (HC), UFPR; ${ }^{2}$ P rofessor Assistente da Disciplina de Radiologia Médica, UFPR; ${ }^{3}$ Acadêmico de Medicina, UFPR; ${ }^{4}$ P rofessor Assistente do De pa rtamento de Pediatria, UFPR; ${ }^{5}$ Médico Residente do Serviço de Pediatria, HC/UFPR; ${ }^{6}$ P rofessor Titular do Departamento de Anatomia Patológica, UFPR.
}

Recebido 10 Agosto 2004, recebido na forma final 10 Fevereiro 2005. Aceito 4 Abril 2005.

Dr. Emerson L. Gasparetto - Avenida Silva Jardim 296 / 502 - 80230-00 Curitiba PR - Brasil. E-mail:gasparetto@hotmail.com 


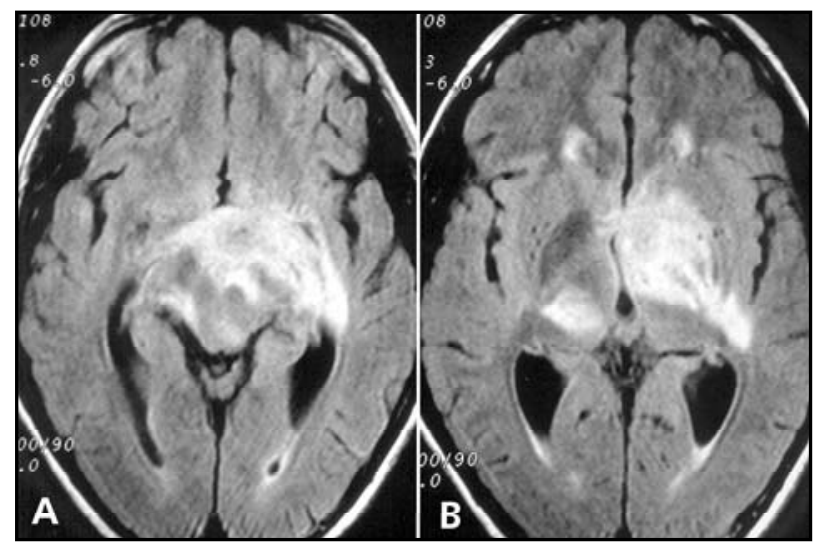

Fig $1 A$ e $B$. Ressonância magnética em cortes axiais ponderados em FLAIR T2 demonstrando lesão expansiva irregular localizada em tálamo, hipotálamo e quiasma óptico à esquerda, com extensa área de edema vasogênico ao seu redor. Evidencia-se também dilatação discreta dos cornos temporais dos ventrículos laterais.

metimento isolado do SNC sob a forma de histoplasmoma em paciente imunocompetente, com ênfase aos achados de imagem.

\section{CASO}

Paciente masculino, 13 anos, apresentando diminuição da acuidade auditiva e visual à esquerda, cefaléia holocraniana e vômitos durante 15 dias. Relatava criação de aves em casa e história de crises convulsivas generalizadas desde os 7 anos de idade, não sendo realizada investigação diagnóstica prévia. Ao exame físico apresentava-se sonolento, com despertar aos chamados, evidenciando-se também redução da acuidade visual e auditiva à esquerda, além de hemiparesia à direita. O eletrencefalograma mostrou alentecimento da atividade de base no hemisfério cerebral esquerdo. $O$ hemograma não a presentava alterações e a análise de líquido cefalorr aquidiano revelou líquor xantocrômico, límpido, com $81 \%$ de linfócitos, glicose $=33 \mathrm{mg} / \mathrm{dl}$, proteínas $=210 \mathrm{mg} / \mathrm{dl}$ e ausência de fungos no estudo micológico direto e culturas.

A ressonância magnética revelou lesão expansiva i rregularmente arredondada, isointensa ao parênquima encefálico em todas as sequências, localizada na topografia do tálamo, hipotálamo e quiasma óptico à esquerda (Figs 1 e 2). A lesão media cerca de 20 mm, apresentava extensa área de edema vasogênico ao seu redor e impregnava-se de forma anelar pelo gadolínio. Observava-se ainda compressão sobre o terc e i roventrículo à esquerda e dilatação discreta dos ventrículos laterais.

O paciente foi submetido a biópsia estereotáxica. A avaliação histológica do material obtido demonstro $u$ tecido cerebral difusamente alterado por processo inflamatório granulomatoso necrotizante, composto por células histiocitárias epitelióides, com ocasionais células gigantes multinucleadas tipo Langerhans. $O$ citoplasma

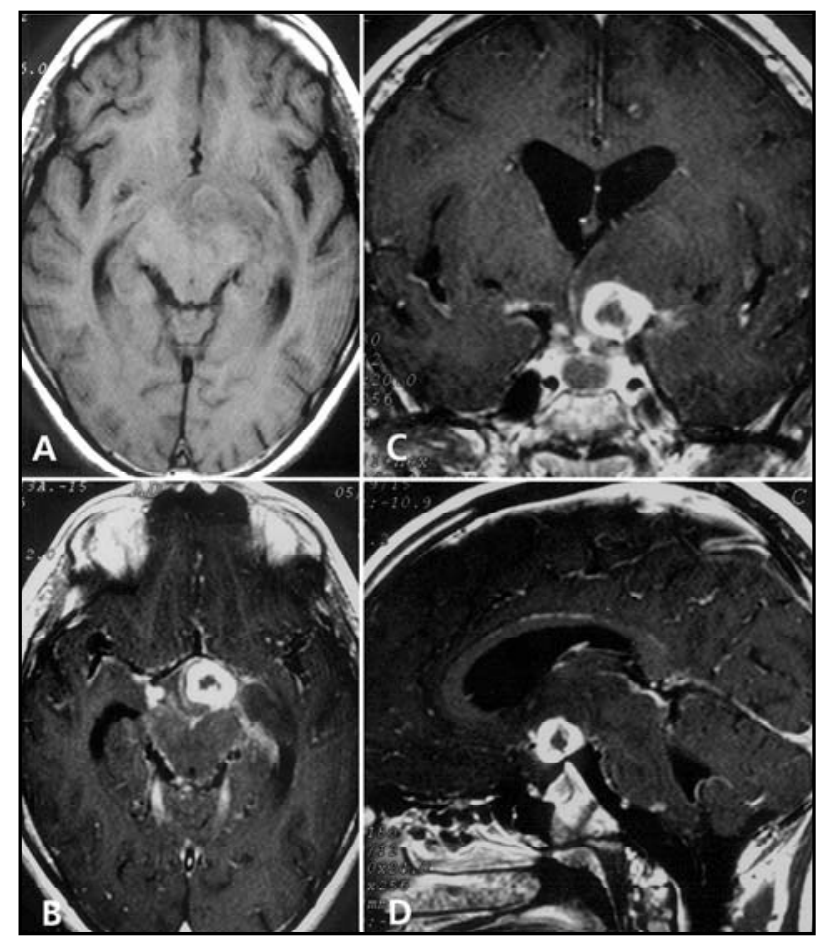

Fig $2 A, B, C$ e $D$. Ressonância magnética em cortes axiais pré$(A)$ e pós-contraste (B), e em cortes coronais (C) e sagitais (D) pós-contraste. Na topografia do tálamo, hipotálamo e quiasma óptico à esquerda evidencia-se lesão expansiva irregulamente arredondada, isointensa ao parênquima encefálico, com impregnação anelar pelo gadolínio. Observava-se ainda discreto realce das meninges adjacentes à lesão.

dessas células continha estruturas arredondadas com cápsula birrefringente e brotamento lateral único, ambos positivos ao PAS com digestão e ao Grocott, sendo definido o diagnóstico de infecção pelo Histoplasma capsulatum (Fig 3). Foi iniciado tratamento com fluconazol e após 6 meses o paciente apresentou melhora clínica, persistindo apenas com discreto déficit visual à esquerda.

\section{DISCUSSÃO}

O Histoplasma capsulatum é um fungo dimórfico capaz de infectar humanos. A inalação de microconídios a partir de solo contaminado, principalmente em locais com excrementos de aves e morcegos, é a principal via de contaminação. A partir desta fase pode ocorrer disseminação hematogênica antes do desenvolvimento de imunidade celular. A doença disseminada é a forma de apresentação mais comum, principalmente em pacientes imunocomprometidos, cursando na maioria dos casos com acometimento pulmonar. No presente caso o paciente referia criação de aves em casa, o que pode ter sido a via de contaminação pelo fungo 4,5 . 


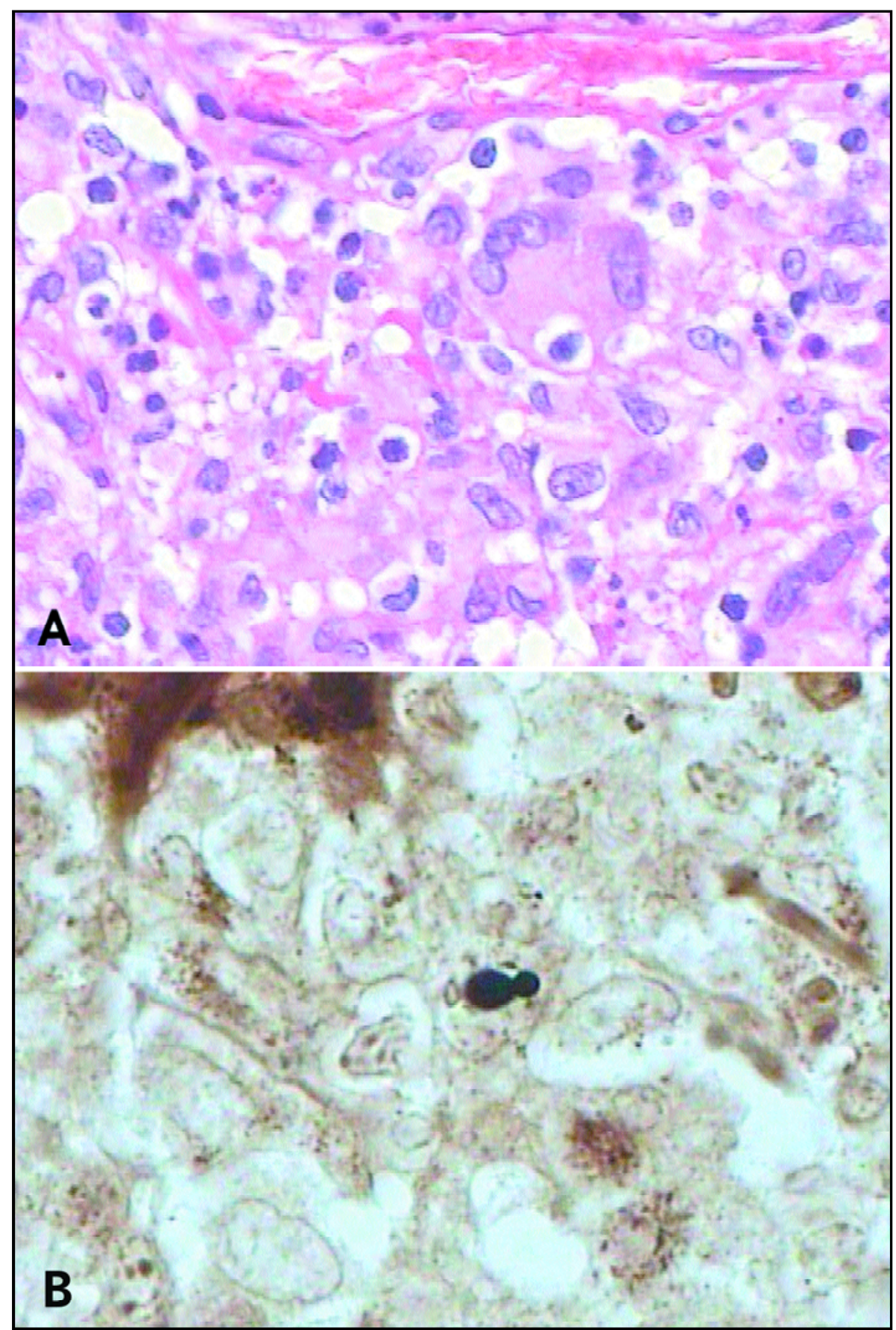

Fig $3 A$ e B. Fotomicrografia demonstra em A, tecido cerebral difusamente alterado por processo inflamatório granulomatoso necrotizante, composto por células histiocitárias epitelióides e ocasionais células gigantes multinucleadas ( $H E \times 40$ ). Em B, observa-se no interior de uma das células histiocitárias epitelióides estrutura arredondada com cápsula birrefringente e brotamento lateral único (Grocott x 100).

O acometimento do SNC pela histoplasmose é raro, em geral ocorrendo sob a forma de doença disseminada. Pacientes com envolvimento isolado do SNC em geral apresentam meningite crônica como principal manifestação. É ainda mais rara a ocorrência da forma tumoral nestes casos. Os histo- plasmomas apresentam-se como lesão cerebral com efeito de massa, podendo ser únicas ou múltiplas, e podem simular uma neoplasia cerebral. Até o presente momento cerca de 14 casos de histoplasmomas no SNC foram descritos na literatura médica mundial. Desses, 11 eram do sexo mas- 
culino, com idade média de 53,5 anos. A maioria dos pacientes $(50 \%)$ apresentava alguma condição $p$ redisponente, como foco pulmonar ativo, diabetes melitus, tuberculose, leucose, mieloma múltiplo, alcoolismo, uso de glicocorticóides e cirrose hepática. O quadro clínico dominante foi de crises convulsivas (6 casos) e de sinais neurológicos focais (6 casos). A localização das lesões era variável, predominando nos hemisférios cerebrais. A maioria foi tratada com uma combinação de ciru rgia e anfotericina $B$, sendo que 5 pacientes evoluíram a óbito ${ }^{6-14}$. A forma tumoral como única manifestação da histoplasmose em paciente imunocompetente é ainda mais rara, havendo apenas um caso descrito na literatura ${ }^{4}$. Este relato descrevia um paciente feminino de 20 anos com história de cefaléia e diplopia e sem evidências de infecção sistêmica. A ressonância magnética demonstrava lesão expansiva com impregnação anelar de contraste, localizada na região talâmica, hipotalâmica e quiasmática à esquerda.

Os achados em exames de imagem em pacientes com histoplasmose acometendo o SNC são inespecíficos. Nos casos de meningite obsenva-se com freqüência impregnação meníngea intensa após a injeção endovenosa de contraste, sem predisposição por uma topografia específica. Nos pacientes que apresentam histoplasmomas como manifestação desta infecção no SNC, em geral a tomografia computadorizada demonstra lesões expansivas hipodensas com captação anelar pelo contraste. A ressonância magnética revela lesões expansivas arredondadas hipointensas nas seqüências ponderadas em $\mathrm{T} 1$ e hiperintensas nas seqüências ponderadas em T2, as quais apresentam captação anelar após a injeção endovenosa de contraste. Áreas de edema vasogênico ao redor das lesões são observadas com freqüência. Este padrão de imagem sugere como diagnósticos diferencias neoplasias primárias e metastáticas, abscessos e contusões cerebrais ${ }^{2,4,11}$.

Concluindo, apesar de manifestação rara da histoplasmose no SNC, os histoplasmomas devem ser incluídos no diagnóstico diferencial de lesões cerebrais expansivas com captação anelar de contraste.

\section{REFERÊNCIAS}

1. Wheat LJ, Batteiger BE, Sathapatayavongs B. Histoplasma capsulatum infections of the central nervous system: a clinical review. Medicine (Baltimore) 1990;69:244-260.

2. Levi GC, Pozzi CM, Hirschheimer SMDS, Chahade WH, Gomes HR, Granato C. Histoplasmose do SNC como única manifestação da doença em hospedeiros imunocompetentes. A rq Neuropsiquiatr 2003;61: 859-863.

3. White HH, Fritzlen TJ. Cerebral granuloma caused by Histoplasma capsulatum. J Neurosurg 1962;19:260-263.

4. Klein CJ, Dinapoli RP, Temesgen Z, Meyer FB. Central nervous system histoplasmosis mimicking a brain tumor: difficulties in diagnostic and treatment. Mayo Clin Proc 1999;74:803-807.

5. Paya CV, Roberts GD, Cockerill FR III. Transient fungemia in acute pulmonary histoplasmosis; detection by new blood-culturing techniques. J Infect Dis 1987;156:313-315.

6. Venger BH, Landon G, Rose JE. Solitary histoplasmomas of the thalamus: case report and literature review. Neuro su rgery 1987;20:784-787.

7. Bridges WR, Echols DH. Cerebellar histoplasmomas: case report. J Neurosurg 1967;26:261-263.

8. Duber RD, Schnitzer M. Histoplasmosis appearing as multiple mass lesions of the brain: report of case. J Am Osteopath Assoc 1982;81: 554-556.

9. LeBourgeois PA. Isolated central nervous system histoplasmosis. South Med J 1979;72:1624-1625.

10. Paphitou NI, Barnett BJ. Solitary parietal lobe histoplasmoma mimicking a brain tumor. Scand J Infect Dis 2002;34:229-232.

11. Vakili ST, Eble JN, Richmond BD, Yount RA. Cerebral histoplasmoma. J Neurosurg 1983;59:332-336.

12. Cooper RA, Goldstein E. Histoplasmosis of the central nervous system: report of two cases and review of the literature. Am J Med 1963;35: 45-57.

13. Greer HD III, Geraci JE, Corbin KB, et al. Disseminated histoplasmosis $\mathrm{p}$ resented as a brain tumor and treated with amphotericin B: report of a case. Mayo Clin Proc 1964;39:490-494.

14. Schochet SS Jr, Sarwar M, Kelly JP, et al. Symptomatic cerebral histoplasmoma: case report. J Neurosurg 1980;52:273-275. 